\title{
Fast Multipole Methods in Service of Various Scientific Disciplines
}

\author{
Levent Gürel \\ Bilkent University Computational Electromagnetics Research Center (BiLCEM) \\ Ankara, TR-06800, Turkey \\ lgurel@gmail.com
}

For more than two decades, several forms of fast multipole methods have been extremely successful in various scientific disciplines. Reduced complexity solutions are obtained for solving different forms of equations that are derived from Maxwell's equations, such as Helmholtz's equation for electrodynamics and Laplace's equation for electrostatics. Fast multipole solvers are developed for and applied to the integral equations derived from Helmholtz's and Laplace's equations. Fast multipole solvers are kernel-dependent techniques, i.e., they rely on certain analytical properties of the integral-equation kernels, such as diagonalizability. Electromagnetics is not the only discipline benefiting from the fast multipole methods; a plethora of computations in various disciplines, such as the solution of Schroedinger's equation in quantum mechanics and the calculation of gravitational force in astrophysics, to name a few, exploit the reducedcomplexity nature of the fast multipole methods. Acoustics, molecular dynamics, structural mechanics, and fluid dynamics can be mentioned as other disciplines served by the fast multipole methods.

Solutions of some of the largest problems reported in the literature during the past decade are obtained with the fast multipole methods, not only very efficiently, but also very accurately. Accuracy is due to the rigor of the integral equations and the error-controllable nature of the multipole solvers. Efficiency of the multipole solvers is due to their ability to take advantage of the underlying rank-deficient blocks of the system matrix.

In this talk, examples of the use of the fast multipole methods in various scientific disciplines will be discussed. 